\title{
CHANGES IN TECHNIQUE OF HANDSPRING DOUBLE SALTO FORWARD TUCKED PERFORMED ON HORSE AND VAULTING TABLE
}

\author{
I. Čuk, S. M. Ferkolj \\ University of Ljubljana, Faculty of Sport, Ljubljana, Slovenia
}

\begin{abstract}
Aim of the research was to determine changes in technique parameters while performing handspring double salto forward tucked (Roche) on old horse and new vaulting table. On a sample of 9 vaults performed in 2000 World Cup in Ljubljana on horse and 9 vaults performed at World championship in Debrecen on vaulting table we made a series of t-tests for biomechanics kinematics parameters. There are differences in many variables, but most important are those related to the support phase (position of hands, take off vertical velocity) which also causes better outcome during the flight and landing. New vaulting table is really much better apparatus than the horse as has better place for support, which makes easier production of angular momentum (inclined table) and higher vertical take off velocity.
\end{abstract}

Key words: artistic gymnastics, vault, horse, table, biomechanics, double salto

\section{INTRODUCTION}

At World Championship 2001 in Ghent FIG (FIG, 2001) changed their tradition and they replaced old horse with vaulting table (Figure 1). After pre tensioned apparatus in ' 50 this is the biggest change in apparatus design. 
By FIG norms vaulting horse is $160 \mathrm{~cm}$ long, $35 \mathrm{~cm}$ wide and 135 high (FIG, 1989). Vaulting table is $95 \mathrm{~cm}$ wide and 95 to $105 \mathrm{~cm}$ long and $135 \mathrm{~cm}$ high. Wider and shorter table is safer [4]. Upper area of the table is slightly inclined (5 degrees). New apparatus has more advantages with wider and slightly inclined support area, what gives gymnast better anatomical support, and better position for arms take off action (Figure 2) [1, 4].
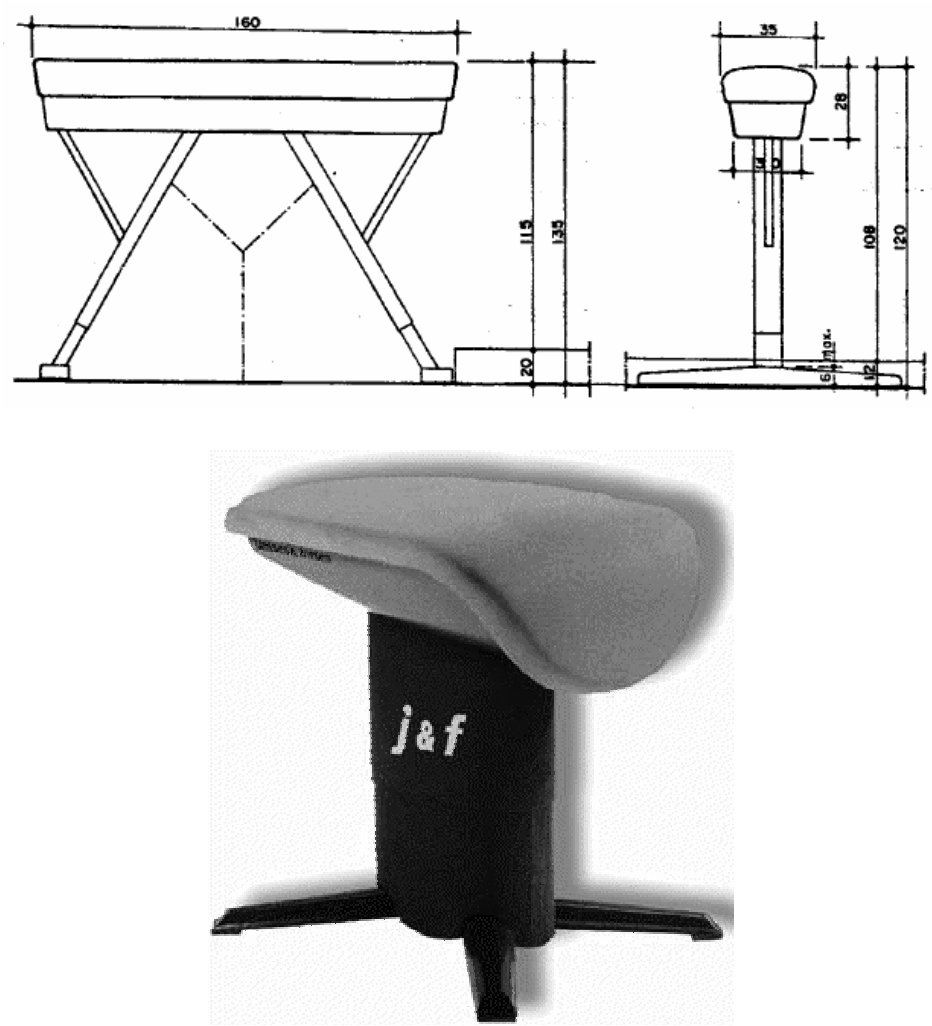

Figure 1. Vaulting horse and vaulting table (FIG, 1989) [2] 

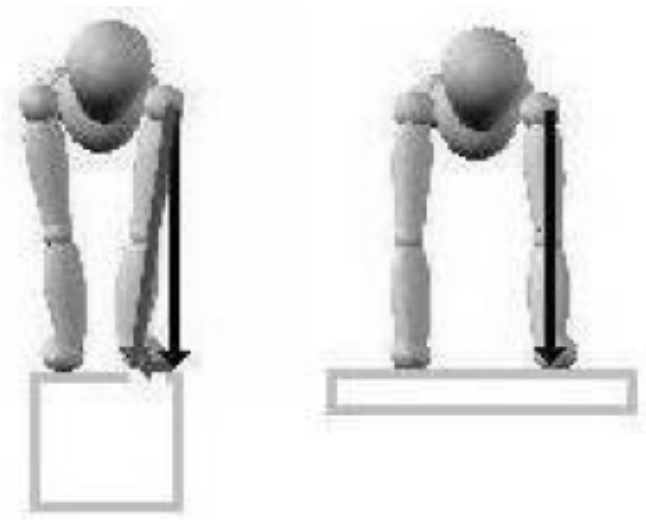

Figure 2. Support position on horse and vaulting table [1]

As we were aware that support is more efficient on new vaulting table, we were searching if beside support are also some other changes in technique of top level vaults and how this change reflects on other biomechanics variables. One of the most difficult jumps nowadays is handspring double salto forward tucked (FIG, 2006), which gymnasts performed on old horse and new vaulting table, within such quantity that we can do statistical analysis.

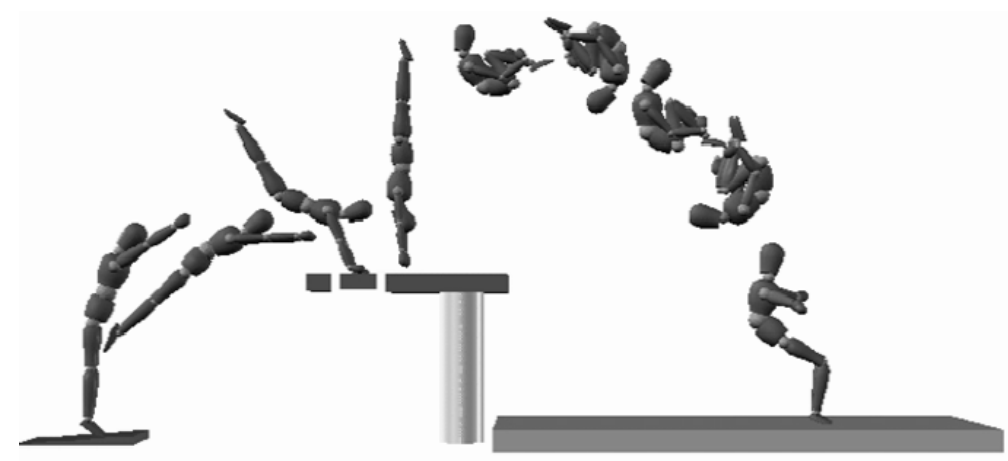

Figure 3. Handspring double salto forward tucked (Roche) [1] 


\section{MATERIALS AND METHODS}

Sample of gymnasts were those gymnasts who have performed handspring and double salto forward tucked at World Cup competition in Ljubljana $2000(\mathrm{~N}=9)$ and those gymnasts who have performed same type of vault at World Championship in Debrecen $2002(\mathrm{~N}=9)$.

Kinematic analysis were done with APAS-Ariel performance analyses system (Ariel Dynamics Inc., San Diego, CA). We used Sušanka, Otahal, Karas [8] 15-segment body model defined with 17 points. All the jumps were recorded during the competition with two orthogonal SVHS cameras with 50 frames per second. All data were smoothed with digital filter of range 7 . We calculated trajectories, velocities, time and angles of important positions in following phases of the vault: support on springboard, the first flight, support on apparatus, the second flight and landing; all together we defined 104 variables.

Statistic analysis were done with SPSS (Statistical Package for the Social Sciences, 12.0, Chicago, IL, USA). First we calculated differencies in quality of jumps between horse and table. Good jump meant jump without fall (on table were 6 and on horse 5 good ones) and bad jump was defined as jump with fall (on table were 3 and on horse 4 bad ones). Calculated $\chi^{2}$ (0,12; non significant differences) showed no differences in quality of jumps. For each variable we calculated descriptive statistics, than F-test between both groups and considering results of F-test we calculated t-test (for equal or unequal variances), only significant differencies in variables are introduced. 


\section{RESULTS AND DISCUSSION}

Table 1. Results of springboard support phase variables

\begin{tabular}{|c|c|c|c|c|c|}
\hline & & Table & Horse & $\mathrm{p}(\mathrm{F})$ & pt-test \\
\hline \multirow{5}{*}{$\begin{array}{l}\text { velocity } \\
\text { BCG Vx } \\
\text { 1. touch } \\
\text { springboard } \\
{[\mathrm{m} / \mathrm{s}]}\end{array}$} & $\mathbf{X}$ & 7.967 & 7.581 & 0.562 & 0.010 \\
\hline & MAX & 8.350 & 7.875 & & \\
\hline & MIN & 7.575 & 7.150 & & \\
\hline & SD & 0.283 & 0.229 & & \\
\hline & SE & 0.188 & 0.169 & & \\
\hline \multirow{5}{*}{$\begin{array}{l}\text { velocity } \\
\text { BCG Vy } \\
\text { 1. touch } \\
\text { springboard } \\
{[\mathrm{m} / \mathrm{s}]}\end{array}$} & $\mathbf{X}$ & 1.113 & 0.784 & 0.674 & 0.038 \\
\hline & MAX & 1.350 & 1.025 & & \\
\hline & MIN & 0.725 & 0.450 & & \\
\hline & SD & 0.236 & 0.200 & & \\
\hline & SE & 0.172 & 0.158 & & \\
\hline \multirow{5}{*}{$\begin{array}{l}\text { velocity } \\
\text { BCG Vxyz } \\
\text { 1. touch } \\
\text { springboard } \\
{[\mathrm{m} / \mathrm{s}]}\end{array}$} & $\mathbf{X}$ & 8.049 & 7.623 & 0.531 & 0.008 \\
\hline & MAX & 8.459 & 7.932 & & \\
\hline & MIN & 7.624 & 7.198 & & \\
\hline & SD & 0.298 & 0.237 & & \\
\hline & SE & 0.193 & 0.172 & & \\
\hline \multirow{5}{*}{$\begin{array}{l}\text { velocity } \\
\text { BCG Vx } \\
\text { last touch } \\
\text { springboard } \\
{[\mathrm{m} / \mathrm{s}]}\end{array}$} & $\mathbf{X}$ & 5.042 & 4.667 & 0.739 & 0.062 \\
\hline & MAX & 5.625 & 5.350 & & \\
\hline & \begin{tabular}{|l|} 
MIN \\
\end{tabular} & 4.525 & 4.250 & & \\
\hline & SD & 0.328 & 0.370 & & \\
\hline & SE & 0.202 & 0.215 & & \\
\hline \multirow{5}{*}{$\begin{array}{l}\text { velocity } \\
\text { BCG Vy } \\
\text { last touch } \\
\text { springboard } \\
{[\mathrm{m} / \mathrm{s}]}\end{array}$} & $\mathrm{X}$ & 4.654 & 4.597 & 0.368 & 0.945 \\
\hline & MAX & 4.725 & 4.925 & & \\
\hline & \begin{tabular}{|l|} 
MIN \\
\end{tabular} & 4.300 & 4.200 & & \\
\hline & SD & 0.138 & 0.192 & & \\
\hline & SE & 0.131 & 0.155 & & \\
\hline \multirow{5}{*}{$\begin{array}{l}\text { velocity } \\
\text { BCG Vxyz } \\
\text { last touch } \\
\text { springboard } \\
{[\mathrm{m} / \mathrm{s}]}\end{array}$} & $\mathbf{X}$ & 6.868 & 6.562 & 0.433 & 0.031 \\
\hline & MAX & 7.346 & 6.875 & & \\
\hline & MIN & 6.475 & 6.351 & & \\
\hline & SD & 0.244 & 0.183 & & \\
\hline & SE & 0.175 & 0.151 & & \\
\hline \multirow{5}{*}{$\begin{array}{l}\text { angle } \\
\text { thrunk / thig } \\
\text { hip }\end{array}$} & $\mathbf{X}$ & 103.0 & 111.9 & 0.910 & 0.005 \\
\hline & MAX & 111.9 & 118.3 & & \\
\hline & MIN & 92.6 & 101.4 & & \\
\hline & SD & 5.9 & 5.7 & & \\
\hline & SE & 0.9 & 0.8 & & \\
\hline
\end{tabular}


Velocity (in xyz) of gymnasts BCG (Body Centre of Gravity) at touch down on springboard jumping on horse is $7.623 \mathrm{~m} / \mathrm{s}$ and $8.049 \mathrm{~m} / \mathrm{s}$ on table, the difference of $0.426 \mathrm{~m} / \mathrm{s}$ is significant Velocity (in xyz) of gymnasts BCG at take off from the springboard jumping on horse is $6.562 \mathrm{~m} / \mathrm{s}$ and $6.868 \mathrm{~m} / \mathrm{s}$ on table, lose of velocity is for both similar (horse $1.162 \mathrm{~m} / \mathrm{s}$ and table $1.172 \mathrm{~m} / \mathrm{s}$ ). At touch down BCG velocity in $\mathrm{x}$ and $\mathrm{y}$ axis is higher for table and also hip angle show more open gymnast position, while at take off persist only the differnce in BCG velocity (in xyz). Diferences in velocities in $\mathrm{x}$ and $\mathrm{y}$ axis are not significant, what shows quite an interesting variance, how gymnasts gain angular momentum on very individual basis. Higher BCG velocity on springboard at touh down and take off table can be explained by famous Fitts law [3,7], which says bigger the area to reach higher velocity can be used; higher velocity means lower control and lover precision. As the horse has smaller support area than table $[1,4,5,6]$ handspring double salto forward tucked is performed with lower BCG velocity on the horse according to Fitts law.

Table 2. Results of support phase variables

\begin{tabular}{|c|c|c|c|c|c|}
\hline & & Table & Horse & $\mathrm{p}(\mathrm{F})$ & pt-test \\
\hline \multirow{4}{*}{$\begin{array}{l}\text { Support } \\
\text { time }\end{array}$} & $\mathbf{X}$ & 0.162 & 0.162 & 0.078 & 1.000 \\
\hline & MAX & 0.180 & 0.200 & & \\
\hline & MIN & 0.140 & 0.140 & & \\
\hline & SD & 0.012 & 0.023 & & \\
\hline [s] & SE & 0.039 & 0.054 & & \\
\hline \multirow{5}{*}{ Hand grip } & $\mathbf{X}$ & 0.439 & 0.176 & 0.012 & 0.000 \\
\hline & MAX & 0.490 & 0.213 & & \\
\hline & MIN & 0.325 & 0.149 & & \\
\hline & SD & 0.054 & 0.020 & & \\
\hline & SE & 0.082 & 0.050 & & \\
\hline \multirow{5}{*}{$\begin{array}{l}\text { Proportion } \\
\text { Shoulders } \\
\text { wide/ } \\
\text { Support wide }\end{array}$} & $\mathbf{X}$ & 0.992 & 2.494 & 0.179 & 0.000 \\
\hline & MAX & 1.314 & 2.822 & & \\
\hline & MIN & 0.859 & 2.127 & & \\
\hline & SD & 0.143 & 0.236 & & \\
\hline & SE & 0.134 & 0.172 & & \\
\hline
\end{tabular}




\begin{tabular}{|c|c|c|c|c|c|}
\hline & & Table & Horse & $p(F)$ & pt-test \\
\hline \multirow{5}{*}{$\begin{array}{l}\text { Velocity } \\
\text { BCG } \\
\mathbf{V x} \\
\text { Take off } \\
{[\mathrm{m} / \mathrm{s}]}\end{array}$} & $\mathbf{X}$ & 3.929 & 3.644 & 0.915 & 0.379 \\
\hline & MAX & 4.675 & 4.375 & & \\
\hline & MIN & 3.225 & 3.200 & & \\
\hline & SD & 0.438 & 0.421 & & \\
\hline & SE & 0.234 & 0.229 & & \\
\hline \multirow{5}{*}{$\begin{array}{l}\text { Velocity } \\
\text { BCG } \\
\text { Vy } \\
\text { Take off } \\
{[\mathrm{m} / \mathrm{s}]}\end{array}$} & $\mathbf{X}$ & 4.146 & 3.803 & 0.601 & 0.005 \\
\hline & MAX & 4.425 & 4.125 & & \\
\hline & MIN & 3.900 & 3.500 & & \\
\hline & SD & 0.183 & 0.222 & & \\
\hline & SE & 0.151 & 0.167 & & \\
\hline \multirow{5}{*}{$\begin{array}{l}\text { Velocity } \\
\text { BCG } \\
\text { Vxyz } \\
\text { Take off } \\
{[\mathrm{m} / \mathrm{s}]}\end{array}$} & $\mathbf{X}$ & 5.724 & 5.278 & 0.492 & $\mathbf{0 . 0 3 7}$ \\
\hline & MAX & 6.235 & 5.819 & & \\
\hline & MIN & 5.257 & 4.743 & & \\
\hline & SD & 0.286 & 0.368 & & \\
\hline & SE & 0.189 & 0.214 & & \\
\hline
\end{tabular}

On the table gymnast has arms almost parallel and orthogonal to support, what is most efficient kind of support. Proportion between shoulders and hand support changed as we expected [1, 4]. Better position of arms gives them position to generate higher take off force what shows out also as higher BCG velocity in y axis. By calculating difference of force between vertical component and horizontal compomponent; vertical component of force on table is $3 \%$ higher. Inclined table $(5 \%)$ rises orthogonal force on table, what gives by reaction force of table better take off results (higher take off force, higher angular momentum [5].

Results of support phase show that support time remained almost identical on vaulting table, therefore it can be considered, as elasticity of new vaulting table was not changed, that 0.162 second is somewhat ideal time for force impact [1].

Significant difference is between velocity at take off from apparatus. Higher vertical velocity is on table, as well as velocity in space. Similar results has found BY Takei [9]. Angles between body segments (head, arms, trunk, legs) has not changed significantly. 
Table 3. Results of flight phase variables

\begin{tabular}{|c|c|c|c|c|c|}
\hline & & Table & Horse & $\mathrm{p}(\mathrm{F})$ & pt-test \\
\hline \multirow{5}{*}{$\begin{array}{l}\text { Angular velocity } \\
\text { From take off } \\
\text { To } \\
\text { 1st salto } \\
\text { [degrees./s] }\end{array}$} & $\mathbf{X}$ & 800.5 & 800.2 & 0.332 & 0.318 \\
\hline & MAX & 822.9 & 830.0 & & \\
\hline & MIN & 728.0 & 767.4 & & \\
\hline & SD & 29.5 & 20.7 & & \\
\hline & SE & 1.9 & 1.6 & & \\
\hline \multirow{5}{*}{$\begin{array}{l}\text { Angular velocity } \\
\text { 1st salto } \\
\text { to } \\
\text { 2nd salto } \\
\text { [degrees. } / s]\end{array}$} & $\mathbf{X}$ & 1104.5 & 1075.2 & 0.584 & 0.972 \\
\hline & MAX & 1200.0 & 1125.0 & & \\
\hline & MIN & 1000.0 & 1000.0 & & \\
\hline & SD & 64.1 & 52.5 & & \\
\hline & SE & 2.8 & 2.6 & & \\
\hline \multirow{5}{*}{$\begin{array}{l}\text { Angular velocity } \\
\text { 2nd salto } \\
\text { to } \\
\text { Touch down } \\
\text { [degrees. } / \text { s] }\end{array}$} & $X$ & 693.2 & 797.7 & 0.412 & 0.032 \\
\hline & MAX & 820.9 & 960.5 & & \\
\hline & MIN & 605.0 & 606.0 & & \\
\hline & SD & 86.0 & 116.2 & & \\
\hline & SE & 3.3 & 3.8 & & \\
\hline \multirow{5}{*}{$\begin{array}{l}\text { Time } \\
\text { Of } \\
\text { flight }\end{array}$} & $\mathbf{X}$ & 1.056 & 1.022 & 0.503 & 0.021 \\
\hline & MAX & 1.080 & 1.060 & & \\
\hline & MIN & 1.000 & 0.980 & & \\
\hline & SD & 0.024 & 0.031 & & \\
\hline & SE & 0.055 & 0.062 & & \\
\hline \multirow{5}{*}{$\begin{array}{l}\text { Time } \\
\text { from take off } \\
\text { to max } \\
\text { contraction } \\
{[s]}\end{array}$} & $\bar{X}$ & 0.230 & 0.258 & 0.010 & 0.027 \\
\hline & MAX & 0.240 & 0.320 & & \\
\hline & MIN & 0.220 & 0.220 & & \\
\hline & SD & 0.011 & 0.029 & & \\
\hline & SE & 0.036 & 0.060 & & \\
\hline \multirow{5}{*}{$\begin{array}{l}\text { Time } \\
\text { from 2nd salto } \\
\text { to } \\
\text { touch down } \\
{[s]}\end{array}$} & $\mathbf{X}$ & 0.247 & 0.209 & 0.055 & 0.005 \\
\hline & MAX & 0.260 & 0.220 & & \\
\hline & MIN & 0.200 & 0.200 & & \\
\hline & SD & 0.022 & 0.011 & & \\
\hline & SE & 0.052 & 0.036 & & \\
\hline \multirow{5}{*}{$\begin{array}{l}\text { Angle } \\
\text { trunk } \\
\text { Thigh } \\
\text { Max. } \\
\text { [degrees] }\end{array}$} & $\mathbf{X}$ & 49.3 & 42.5 & 0.864 & 0.023 \\
\hline & MAX & 57.6 & 53.1 & & \\
\hline & MIN & 42.5 & 33.2 & & \\
\hline & SD & 5.9 & 5.5 & & \\
\hline & SE & 0.9 & 0.8 & & \\
\hline
\end{tabular}




\begin{tabular}{|l|l|r|r|r|c|}
\hline & & Table & Horse & $\mathrm{p}(\mathrm{F})$ & pt-test \\
\hline \multirow{2}{*}{$\begin{array}{l}\text { Angle } \\
\text { Thigh } \\
\text { Calf } \\
\text { Max. }\end{array}$} & X & $\mathbf{6 0 . 4}$ & $\mathbf{4 9 . 3}$ & 0.437 & $\mathbf{0 . 0 0 1}$ \\
\cline { 2 - 4 } [degrees] & MAX & 69.2 & 58.3 & & \\
\cline { 2 - 4 } & MIN & 54.1 & 40.4 & & \\
\cline { 2 - 4 } & SD & 4.8 & 6.3 & & \\
\cline { 2 - 4 } & SE & 0.8 & 0.9 & & \\
\hline
\end{tabular}

Higher vertical take off force on table is reason for higher peak BCG height in the second flight, higher is BCG after finishing the first and the second salto on table. In a whole the time for the second flight is on table longer.

On the table is also faster bending from the take off up to the maximum tuck position in salto. Reason is because angles on horse are smaller, therefore gymnast on table is more open (moment of inertia is higher). Similar results were obtained also by Takei [9].

Surprisingly angular velocity during first and second salto is for both same, however hip and knee angles during salto are significantly different, as during vaults from vaulting table gymnasts are more open, what means, that during flight phase they have higher angular momentum $[5,10]$.

On table is extended time from finished second salto to the first contact at landing, as BCG height after the second salto on horse is $2.07 \mathrm{~m}$ and on table $2.29 \mathrm{~m}$. With higher BCG position and with stretching prior the landing gymnast on table lowers angular velocity what gives him better chances to control landing.

Hip and knee angles at the moment of first touch down are higher on table (gymnasts is more open). Also on table BCG is in moment of touch down higher for $0.12 \mathrm{~m}$.

New vaulting table allows gymnast to gain higher runway velocity, better anatomic-functional position of arms, and therefore higher vertical velocity from apparatus and angular momentum (inclined table), what results in longer time of flight, higher amplituded of flight and better position to prepare for landing $[1,5]$. 
Table 4. Results of landing variables

\begin{tabular}{|c|c|c|c|c|c|}
\hline \multirow{6}{*}{$\begin{array}{l}\text { Angle } \\
\text { Trunk } \\
\text { Thigh } \\
\text { Touch down } \\
\text { [degrees] }\end{array}$} & & Table & Horse & $\mathrm{p}(\mathrm{F})$ & pt-test \\
\hline & $\mathbf{X}$ & 137.7 & 106.6 & 0.731 & 0.006 \\
\hline & MAX & 165.0 & 147.1 & & \\
\hline & MIN & 98.5 & 85.2 & & \\
\hline & SD & 22.2 & 19.6 & & \\
\hline & SE & 1.7 & 1.6 & & \\
\hline \multirow{5}{*}{$\begin{array}{l}\text { Angle } \\
\text { Thigh } \\
\text { Calf } \\
\text { Touch down } \\
\text { [degrees] }\end{array}$} & $X$ & 133.0 & 108.7 & 0.441 & 0.009 \\
\hline & MAX & 152.4 & 135.9 & & \\
\hline & MIN & 94.1 & 88.8 & & \\
\hline & SD & 19.6 & 14.8 & & \\
\hline & SE & 1.6 & 1.4 & & \\
\hline
\end{tabular}

From our results we can conclude that new vaulting table significantly changed performances of gymnasts. However there might be also a catch. Our investigation was performed in time, where there was not a lot gymnast who can perform such vault and accommodation to the new vaulting table was not so world wide spread, and only best gymnasts were performing handspring double salto forward tucked. As gymnast easier gains during the support higher angular momentum, this can be dangerous for those gymnasts who are not physically, technically and mentally prepared for such a difficult vault as new vaulting table gives them blind self confidence.

\section{REFERENCES}

1. Čuk I., Karacsony I. (2004) Vault: methods, ideas, curiosities, history. Ljubljana, ŠTD Sangvinčki.

2. Jenssen \& Fritsen (2003) The vault of the next generation. Netherlands. www.gymmedia.com/jenssen-fritsen/.

3. Magill R. A. (1998) Motor learning - Concepts and Applications. Louisiana, WCB McGraw-Hill, 5th edition.

4. McNeal J. R. (2003) Some guidelines on the transition from the old horse to the new table. USA, Esteren Washington University.

5. Prassas S. (2002) Vaulting mechanics. USA, Colorado State University. 
6. Rand T. (2003) New vaulting table. USA Gymnastics. (www.usagymnastics.org)

7. Schmidt R. A. (1999) Motor control and learning: A behavioral emphasis. Failure analysis associates, Inc. And University of California, Los Angeles.

8. Sušanka P., Otahal S. Karas V. (1987) Zaklady biomechanicky telesnyh cvičeni. Praha, Universita Karlova.

9. Takei Y. (2007) The Roche vault performed by elite gymnasts: Somersaulting technique, deterministic model and judges scores. J. Appl. Biomech. 23, 1-11.

10. Winter A. D. (1990) Biomechanics and motor control of human movement. Waterloo, University of Waterloo.

\section{ACKNOWLEDGEMENTS}

We would like to thanks Ministry of Higher Education and Science for supporting program Kinesiology of monostructural, polystructural complex and conventional sports. Thanks also to International Gymnastics Federation, Hungarian Gymnastics Federation and Slovenian Gymnastics Federation for allowing us to do experiment during competitions.

\section{Corresponding author:}

Ivan Čuk

University of Ljubljana

Faculty of Sport

Gortanova 22

1000 Ljubljana

Slovenia 